\title{
Power Savings with all SiC Inverter in Electric Traction applications
}

\author{
B.K. Chakravarthy ${ }^{1}$, and $G$. Sree Lakshmi ${ }^{2}$ \\ ${ }^{1}$ Research Scholar, Department of Electrical Engineering, University College of Engineering, Osmania University \\ ${ }^{2}$ Member, IEEE
}

\begin{abstract}
The advantage of Silicon Carbide $(\mathrm{SiC})$ based devices are less thermal management requirements and smaller passive components which result in higher power density. SiC devices have higher blocking voltages, lower on-state resistance and switching losses and higher thermal conductivity and operating temperatures. SiC devices can operate at higher voltages, higher frequencies and higher junction temperatures than comparable $\mathrm{Si}$ devices, which results in significant reduction in weight and size of the power converter and increase in system efficiency.
\end{abstract}

\section{Introduction}

The performance and efficiency of traction systems was improved using high efficiency induction motors and has been further enhanced by advances in power devices. Inverters and other traction systems become smaller by using low-loss power devices. Low-loss power devices use Silicon Carbide ( $\mathrm{SiC}$ ) instead of Silicon ( $\mathrm{Si}$ ). $\mathrm{SiC}$ has approximately 10 times the critical breakdown strength of Silicon. The thickness of drift layer in $\mathrm{SiC}$ is approximately one-tenth of that in $\mathrm{Si} \mathrm{[6].} \mathrm{This} \mathrm{allows} \mathrm{for}$ dramatic reduction in conductivity loss and switching loss in $\mathrm{SiC}$ devices [9]. SiC has three times the band-gap width of Si which prevents the flow of leakage current and enables operation at high temperatures.

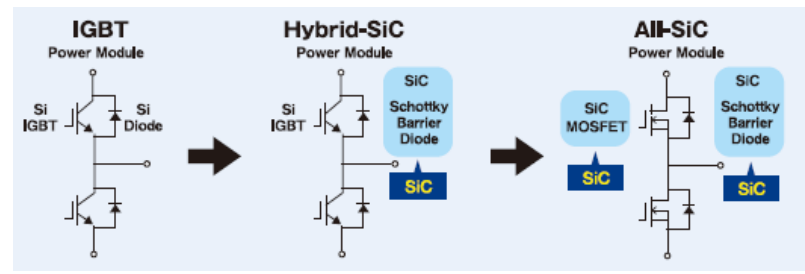

Fig. 1. Evolution in devices

\subsection{Hybrid SiC and All SiC}

\subsubsection{High speed switching operation}

A Hybrid - SiC module is shown in figure 1 It uses Schottky Barrier Diodes (SBDs). High speed switching can be realized with SBDs as they don't have accumulation carriers. The all $\mathrm{SiC}$ module is very compact and has high speed switching capability [1]. It is possible to reduce the size of additional components like reactors due to high speed switching.

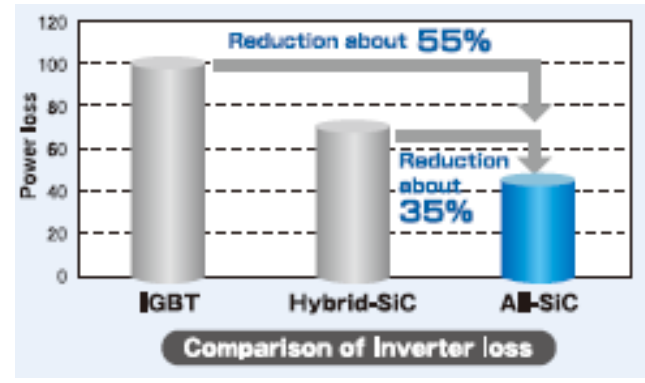

Fig. 2. Power savings in all $\mathrm{SiC}$ device.

\subsubsection{Reduction in power loss}

SiC IGBT modules with SBDs when used with active gate control techniques can reduce the inverter losses by about 35\% [2]. To increase the efficiency of drive system in traction applications, the area of regenerative braking is to be expanded by minimising the use of pneumatic brake. Losses due to harmonics in induction motors can be reduced by optimizing induction motor design and PWM control techniques.

Figures 3 and 4 show the braking force and power fed back during regenerative braking of a locomotive using a conventional IGBT based inverter and those for a twolevel inverter using all $\mathrm{SiC}$ power modules. Table 1 compares the losses in conventional IGBT modules and all $\mathrm{SiC}$ based modules. Figures 5 compares the turn-on characteristics and Figure 6 compares the turn-off characteristics of $\mathrm{SiC}$ and IGBT. The reduction in losses leads to reduction in the size of cooling systems. Traction inverters with all $\mathrm{SiC}$ modules are therefore smaller and lighter.

\footnotetext{
* Corresponding author: B.K. Chakravarthy
} 


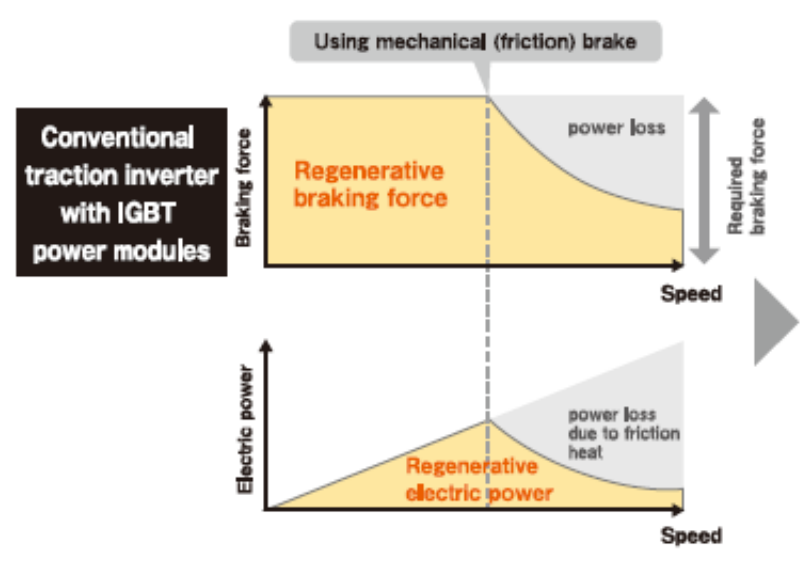

Fig. 3. Regenerative braking with IGBT modules

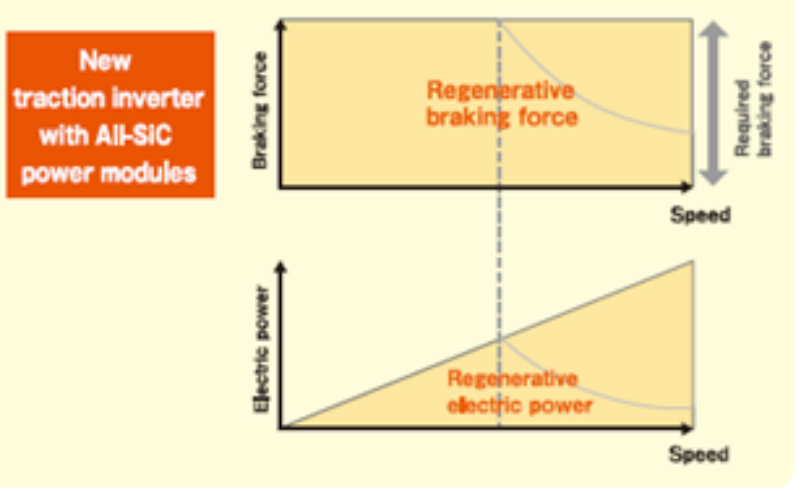

Fig. 4. Regenerative braking with $\mathrm{SiC}$ module

Table 1. Difference in switching of SiC and IGBT

\begin{tabular}{|c|c|c|}
\hline & SiC & IGBT \\
\hline $\begin{array}{c}\text { Turn -on delay } \\
\text { time (ns) }\end{array}$ & 45 & 120 \\
\hline $\begin{array}{c}\text { Turn - on rise time } \\
\text { (ns) }\end{array}$ & 50 & 80 \\
\hline $\begin{array}{c}\text { Turn - off delay } \\
\text { time (ns) }\end{array}$ & 170 & 450 \\
\hline $\begin{array}{c}\text { Turn - off fall time } \\
\text { (ns) }\end{array}$ & 60 & 350 \\
\hline $\begin{array}{c}\text { Reverse recovery } \\
\text { time (ns) }\end{array}$ & 30 & 150 \\
\hline $\begin{array}{c}\text { Turn - on loss (mJ) } \\
\text { Turn -off loss (mJ) }\end{array}$ & 1.7 & 10 \\
\hline Recovery loss (mJ) & 0.1 & 8.5 \\
\hline
\end{tabular}

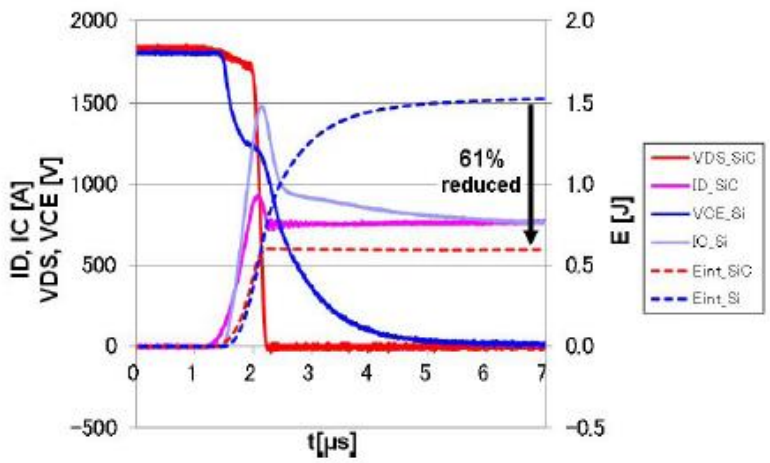

Fig. 5. Turn-on characteristics

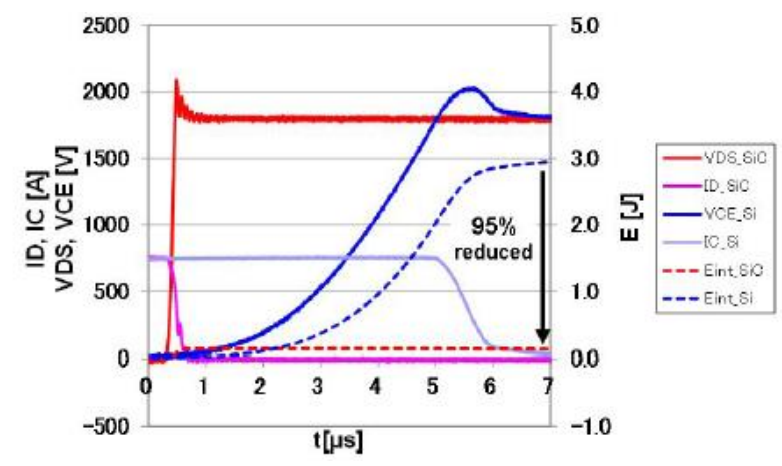

Fig. 6. Turn-off characteristics

\section{Reduction in inverter losses}

Losses can be reduced by as much as $35 \%$ when using all $\mathrm{SiC}$ based modules. The reduction in losses is more predominant [3] as the switching frequency increases as shown in figure 7.

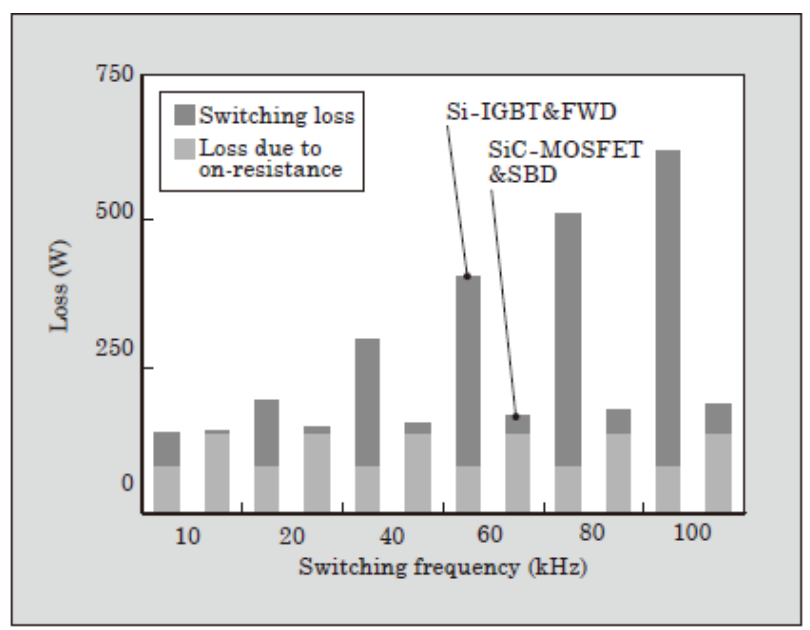

Fig. 7. Variation of losses with switching frequency

The inverter usually switches between three techniques of PWM depending on the speed. A combination of synchronous and asynchronous PWM can be used in the low and medium speed range. The control range for asynchronous PWM can be expanded for inhibiting lower order harmonics through optimization of carrier 
frequency. Low distortion synchronous PWM control can be used to reduce current harmonics in the upper end of medium speed range. In the high-speed range, pulse traction system can be used to reduce lower order harmonics. These techniques are shown in figure 8 .

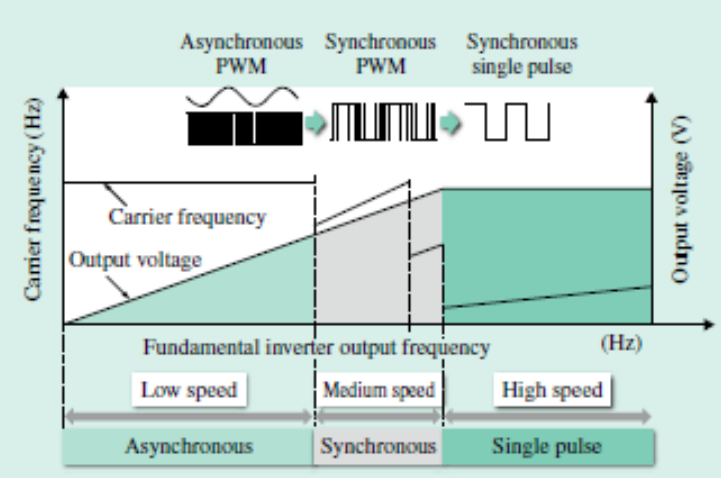

(a) Running profile using conventional PWM control technique

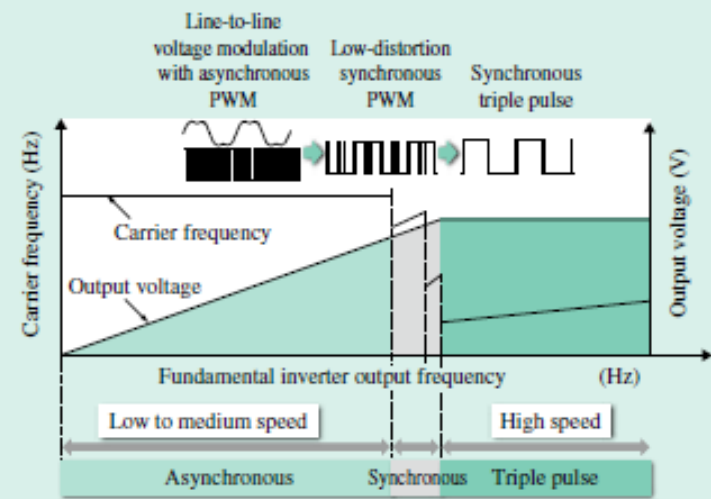

(b) Running profile using new energy-efficient PWM control technique

Fig. 8. Modulation techniques used with traction converter

\subsection{Application in Metro trains}

Metro train in an Electric Multiple Unit (EMU). EMU is an example of distributed traction system. Each unit typically has three cars. Multiple units can be added or removed depending on operating requirements as shown in figure 9.

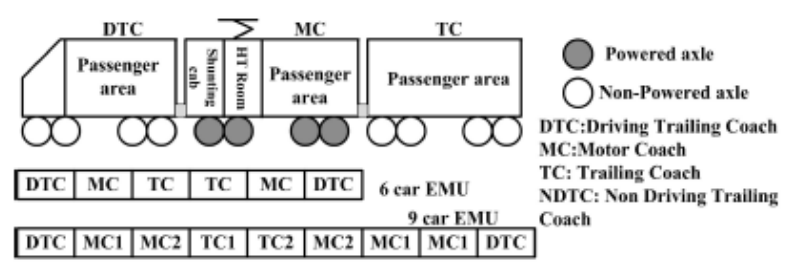

Fig. 9. Electric Multiple Unit (EMU)

Typical power architecture of EMU [2] is shown below in figure 10.

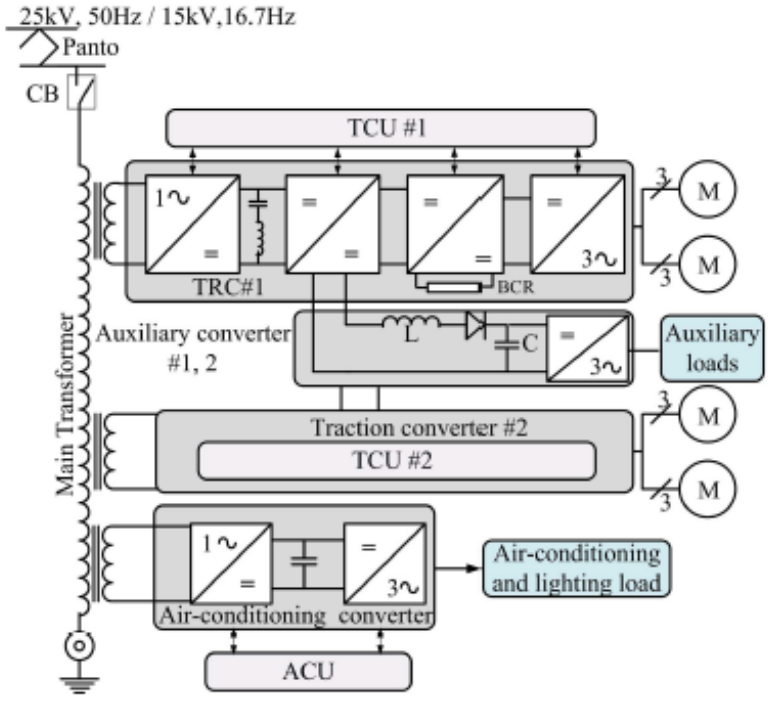

Fig. 10. EMU architecture

When $\mathrm{SiC}$ modules are used in inverter the reduction in the size of the inverter [4] [8] [10] is shown in the figure 11 below. There is also a significant reduction in weight.

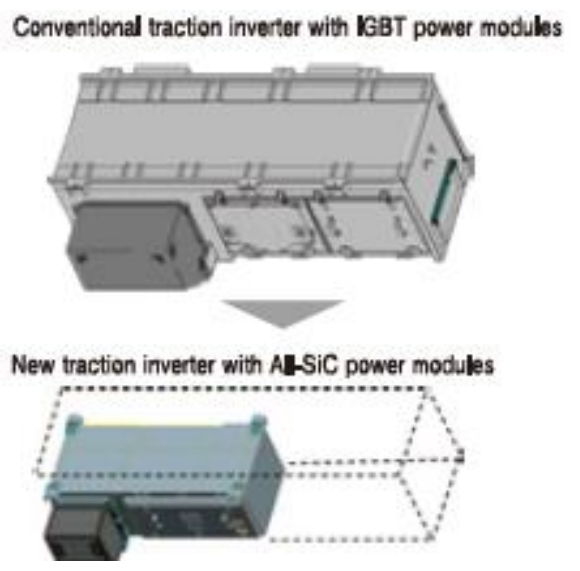

Fig. 11. Size reduction in traction converter

Losses and efficiency are compared for $80 \mathrm{KW}$ traction inverter: [5] [7]

Topology: Three phase inverter

Synchronous rectification ( $\mathrm{SiC}$ version)

DC-link voltage: 400V DC

Current 480Arms (peak) 230Arms (nom)

Switching frequency: $16 \mathrm{kHz}$

$\mathrm{Vgs}=+20 \mathrm{~V} /-5 \mathrm{~V}$ for $\mathrm{SiC}, \mathrm{Vge}= \pm 15 \mathrm{~V}$ for IGBT

Cos(phi): 0.8

Modulation index (MI): 1 
Inverter losses vs \%load

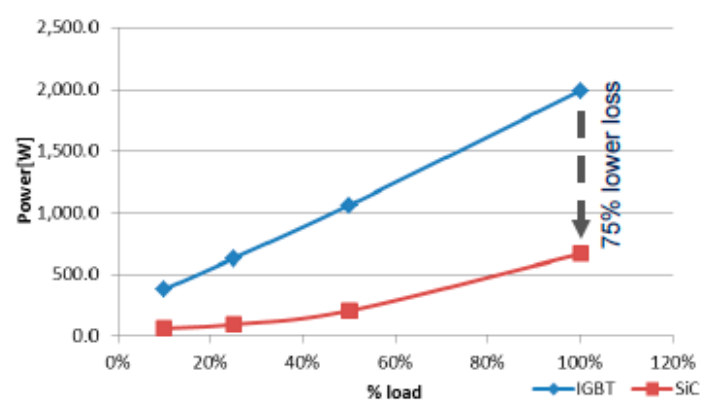

Fig. 11. Comparison of inverter losses

\section{Inverter efficiency vs \%load}

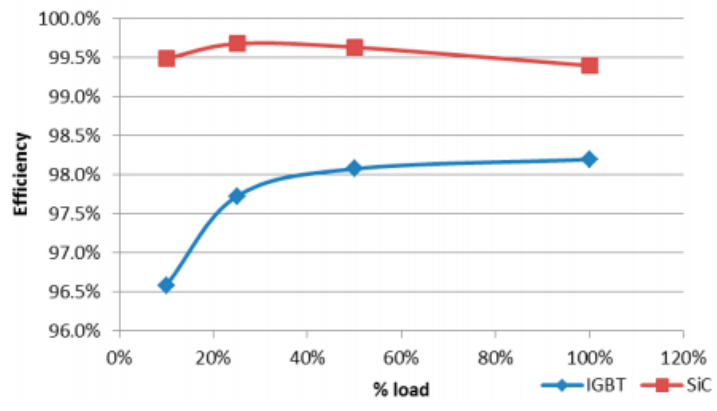

Fig. 12. Comparison of inverter efficiency

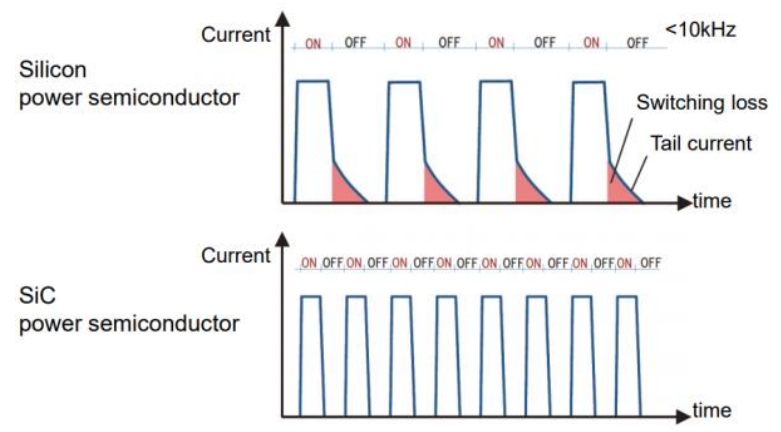

Fig. 13. Low switching loss illustration in $\mathrm{SiC}$

The current waveforms in figure 13 clearly illustrate the low switching loss. As shown in Figure 14, low losses even in high frequency operation helps in reducing the size of the inverter and improves efficiency.

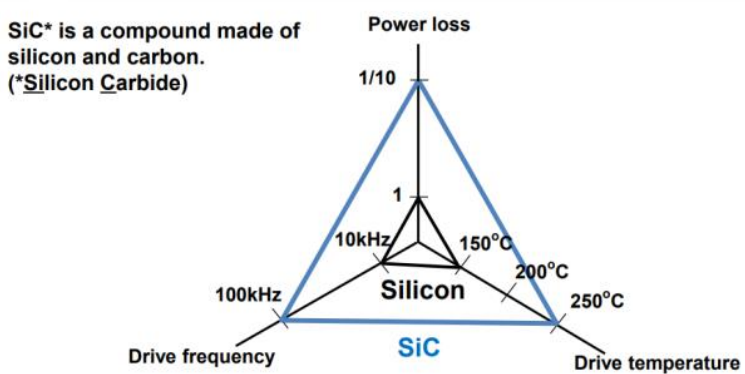

Fig. 14. Improved efficiency by using $\mathrm{SiC}$

\section{Conclusion}

Silicon Carbide ( $\mathrm{SiC}$ ) based devices will have a large impact on inverter efficiency and thermal management issues in traction applications. $\mathrm{SiC}$ has clear operational advantage over conventional devices. SiC based inverters are also being developed for use in Electric Vehicle and Hybrid Electric Vehicle (HEV) applications. These inverters help in increasing the range of the vehicle and in reducing the weight of the vehicle.

\section{References}

1. K. Ishikawa, K. Terasawa, T. Sakai, S. Sugimoto, T. Nishino, Development of rolling stock inverters using SiC, Hitachi Review. 66, 2 (2017)

2. D. Ronanki, S.A. Singh, S.S. Williamson, Comprehensive Topological Overview of Rolling Stock Architecture and Recent Trends in Electric Railway traction Systems. IEEE Trans. on Transportation Electrification, 3, 3 (2017)

3. N. Hayato, H. Yuichiro, $H$. Masafumi. Ultracompact, High-reliability, All-SiC Module, Fuji Electric Review, 59, 4 (2013)

4. www.mitsubishielectric.com

5. www.st.com

6. P. Shamsi, M.McDonough and B. Fahimi, WideBandgap Semiconductor Technology: Its impact on the electrification of the transportation industry., in IEEE Electrification Magazine,1, 2 (2013).

7. M. M. Swamy, Jun-Koo Kang, K. Shirabe, Power Loss, System Efficiency, and Leakage Current Comparison Between Si IGBT VFD and SiC FET VFD With Various Filtering Options, in IEEE Transactions on Industry Applications, 51, 5 (2015).

8. Z. Zhang, F. Wang, L. M. Tolbert, B. J. Blalock and D. J. Costinett, Evaluation of Switching Performance of SiC Devices in PWM Inverter-Fed Induction Motor Drives, in IEEE Transactions on PowerElectronics, 30, 10 (2015).

9. I. Josifović, J. Popović-Gerber and J. A. Ferreira, Improving $\mathrm{SiC}$ JFET Switching Behavior Under Influence of Circuit Parasitics, in IEEE Transactions on Power Electronics, 27, 8 (2012).

10. A. Merkert, T. Krone and A. Mertens, Characterization and Scalable Modeling of Power Semiconductors for Optimized Design of Traction Inverters with $\mathrm{Si}$ - and SiC-Devices, in IEEE Transactions on Power Electronics, 29, 5 (2014). 